\title{
Die Arbeitgeber des öffentlichen Sektors: Institutionelle Stabilität und sozio-ökonomischer Wandel
}

Über die Arbeitsbeziehungen des öffentlichen Sektors ist trotz seiner Größe und Bedeutung für die Gesamtwirtschaft in der Öffentlichkeit wenig bekannt. Insbesondere die Interessen, Organisations- und Verhandlungsstrukturen der öffentlichen Arbeitgeber bleiben unbeachtet. Der Beitrag geht der Frage nach, welche Konsequenzen organisatorische Veränderungen, sozio-ökonomischer Wandel sowie die Privatisierungen von Bahn und Post für die Stabilität der Arbeitsbeziehungen und damit die Arbeits- und Lebensbedingungen der Beschäftigten haben.

BERNDT KELLER

\section{Einleitung}

Sozial- und wirtschaftswissenschaftliche Analysen von Arbeitsbeziehungen konzentrieren sich in Deutschland traditionell wie aktuell auf die Privatwirtschaft und vernachlässigen den öffentlichen Sektor. Diese Unausgewogenheit ist aus mehreren Gründen problematisch. Zum einen ist der öffentliche Sektor der größte Arbeitgeber (von ca. 4,6 Mio. Beschäftigten), zum andern verbieten seine rechtlich-institutionellen Besonderheiten eine Übertragung von Erkenntnissen aus der Privatwirtschaft und erfordern eine eigenständige Analyse.

Insbesondere Arbeitgeberverbände werden in der Forschung selten behandelt (aktuelle Ausnahmen: Schroeder/ Weßels 2010; Behrens 2011). Dieser Befund gilt in besonderem Maße für den öffentlichen Sektor, allerdings auch mit Blick auf die Privatwirtschaft. Auch diese Vernachlässigung ist problematisch, weil die Verbände die Kollektivverhandlungen führen und dadurch Entgelte und übrige Beschäftigungsbedingungen der Tarifbeschäftigten direkt, die der Beamten indirekt beeinflussen.

Bei der Analyse der Arbeitgeberverbände sind folgende rechtlichen und organisatorischen Besonderheiten wesentlich:

(1) Der traditionelle Dualismus der Dienstverhältnisse bedeutet die strikte, im Grundgesetz (Art. 33 GG) vorgegebene Trennung in Beamte und Tarifbeschäftigte. Diese rechtliche Unterscheidung hat zur Folge, dass die Organi- sationsdomänen der Arbeitgeberverbände ${ }^{1}$ ausschließlich die zuletzt genannte Statusgruppe umfassen.

(2) Wirtschaftsverbände bestehen nicht, sodass die in der Privatwirtschaft charakteristische Arbeitsteilung zwischen allgemeinen Wirtschafts- oder Unternehmens- und speziellen Arbeitgeberverbänden bzw. zwischen Wirtschafts- und Sozialpolitik keine Bedeutung hat; auch sog. Mischverbände kommen im Gegensatz zur Privatwirtschaft nicht vor.

(3) Die Arbeitgeberverbände sind weder auf nationaler ${ }^{2}$ noch auf supranationaler ${ }^{3}$ Ebene Mitglied der intersektoralen Dachverbände; ${ }^{4}$ sie bleiben eigenständig-unabhängige Akteure, die jedoch informelle Kontakte zu Verbänden der Privatwirtschaft unterhalten.

\footnotetext{
1 Organisations- bzw. Repräsentationsdomänen stecken den spezifischen Mitgliedschafts- und damit Aufgabenbzw. Zuständigkeitsbereich ab. Unterschiede in den Anforderungen und Erwartungen an die Organisation ergeben sich u. a. aus Besonderheiten der Ebenen (Bund, Länder, Gemeinden/Gemeindeverbände).

2 Bundesvereinigung der Deutschen Arbeitgeberverbände (BDA), http://www.arbeitgeber.de/www/arbeitgeber.nsf/ID/ home.

3 BusinessEurope, http://www.businesseurope.eu/Content/ Default.asp?

4 Mitgliedschaft besteht im European Centre of Enterprises with Public Participation and of Enterprises of General Economic Interest, http://www.ceep.eu/.
} 
(4) Internationale Vergleiche (Bach et al. 1999; European Foundation 2011; European Commission 2013) zeigen, dass derartige Arbeitgeberverbände nicht in allen EUMitgliedsländern bestehen; insofern stellen die deutschen Verbände nicht den Regelfall dar. Häufig übernehmen unabhängige öffentliche Agenturen oder die Regierung bzw. ihre Ministerien Aufgaben der Interessenvertretung.

(5) Last, but not least besteht eine wesentliche Rahmenbedingung in der Tatsache, dass seit den frühen 1990er Jahren die Zahl der Beschäftigten um ca. ein Drittel reduziert wurde (Abbildung 1), woraus ein „schlanker Staat“ bzw. eine „schlanke Verwaltung“ resultiert (Keller 2010a; Vesper 2012). Die Zahl der Tarifbeschäftigten nahm relativ stärker ab als die der Beamten, wodurch die Organisationsdomänen der Verbände kleiner wurden. Im Gegensatz zu diesem seit den frühen 1990er Jahren zu beobachtenden Trend nehmen in den vergangenen Jahren die Beschäftigtenzahlen, vor allem auf kommunaler Ebene, leicht zu (VKA 2012). Diese insgesamt geringen Zuwächse sind vor allem in unterbesetzten Bereichen wie Erziehung und Kindergärten zu verzeichnen (Keller 2013a).

\section{Problemstellung}

Im Folgenden liefere ich mit der Füllung der oben skizzierten Forschungs- und Informationslücke einen Beitrag zu den Arbeitsbeziehungen im öffentlichen Sektor (public sector industrial relations). Ziel ist dabei nicht eine historische (McPherson 1971) oder juristische, sondern eine empirisch orientierte Analyse aktueller Entwicklungen und ihrer Konsequenzen. Gewerkschaften finden nur insoweit Berücksichtigung, als sie zum Verständnis der Strukturen und Aktivitäten von Arbeitgeberverbänden notwendig sind; insofern erfolgt eine Schwerpunktverlagerung im Vergleich zu vorliegenden, auf die Arbeitnehmerseite fokussierten Analysen (Keller 2010a, 2013b). Der Beitrag konzentriert sich aus arbeitsökonomischen Gründen auf die sektorale Ebene und klammert die in dualen Systemen der Arbeitsbeziehungen relevante Betriebs- bzw. Dienststellenebene explizit aus (Keller/Schnell 2003, 2005). Referenz- und Vergleichspunkte sind gelegentlich Entwicklungen in der Privatwirtschaft, vor allem aber in den öffentlichen Sektoren anderer Länder der Europäischen Union (EU) bzw. der Organisation für wirtschaftliche Zusammenarbeit und Entwicklung (OECD).

Der Beitrag skizziert aus Informationsgründen zunächst die weitgehend unbekannten Organisations- und Verhandlungsstrukturen der Arbeitgeberverbände (Abschnitt 3). Anschließend behandelt er detailliert die in den vergangenen beiden Jahrzehnten eingetretenen erheblichen organisatorischen Veränderungen bzw. sozio-ökonomischen Wandlungsprozesse, vor allem die vertikalen und horizontalen Differenzierungen sowie die Privatisierungsmaßnah-
ABB. 1

\section{Entwicklung der Beschäftigung im öffentlichen Dienst*}

Angaben in Mio.
Bund
Länder
Deutsche Bahn ${ }^{1}$
Deutsche Bundespost ${ }^{2}$
Gemeinden/Gemeindeverbände unmittelbarer öffentl. Dienst

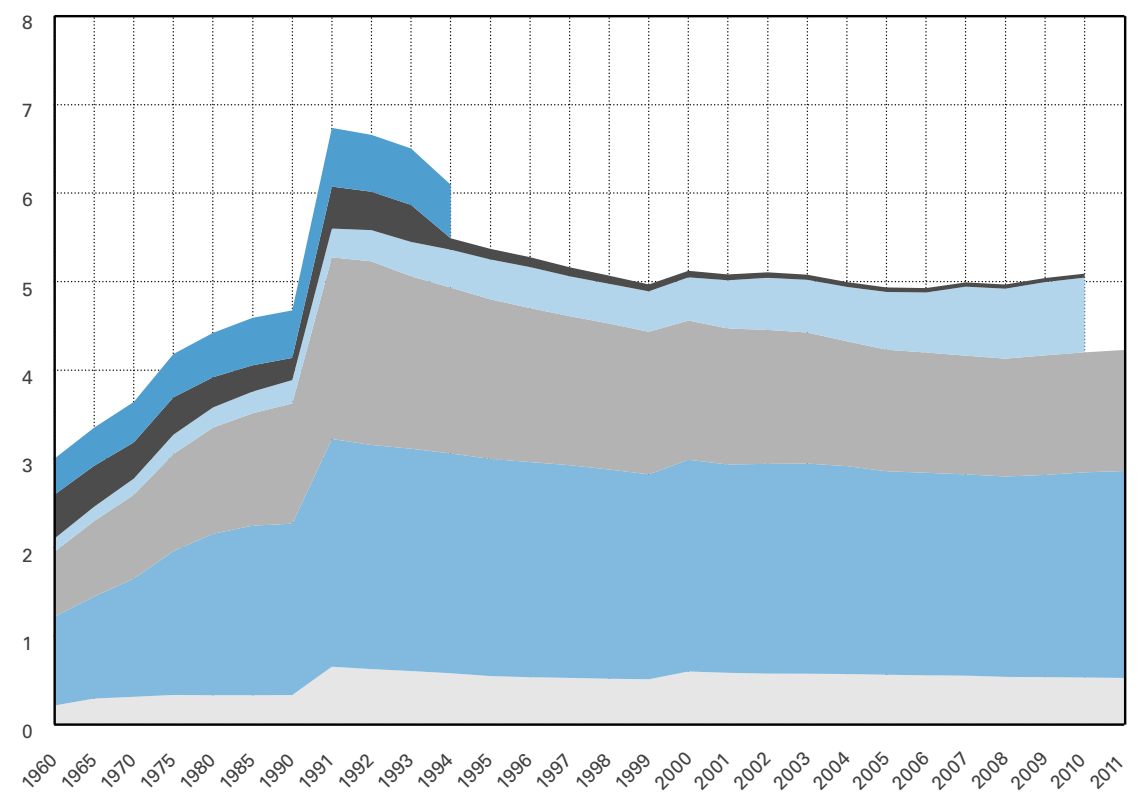

*Zahlen in Mio. bezogen auf den 30. Juni des jeweiligen Jahres.

Bis 1990: Westdeutschland; ab 1991: Deutschland.

Seit 2000: Veränderung des Konzepts zur Zählung des unmittelbaren öffentlichen Dienstes.

Differenzen aufgrund von Rundungen möglich.

'Seit 1995: nicht mehr im öffentlichten Dienst wegen Privatisierung. 2011: n/a

${ }^{2}$ Seit 1994: nicht mehr im öffentlichen Dienst. 2011: n/a

Anmerkung: Das Statistische Bundesamt hat in dem langen, hier relevanten Berichtszeitraum mehrfach das Konzept zur Veröffentlichung seiner Personalstandsstatistik verändert (zuletzt 2012, Ausgliederung von öffentlichten Aufgaben aus der Kernverwaltung der Gebietskörperschaften in rechtlich selbstständige Einrichtungen). Die hier präsentierte Tabelle legt soweit wie möglich Wert auf Konsistenz der Erfassung (auf Basis der bis 2011 geltenden Verfahren). Dennoch sind nicht alle Daten direkt vergleichbar; offizielle Rückrechnungen erfolgen aktuell nur für die 2000er Jahre.

Quelle: Statistisches Bundesamt, Fachserie 14, Reihe 6, verschiedene Jahrgänge; Berechnungen und Darstellung des Autors. men von Bahn und Post. Der Beitrag gibt Antworten auf die Frage nach deren Folgen für die Organisation der Interessen von Arbeitgebern sowie für die institutionelle Stabilität der Arbeitsbeziehungen (4). Den Schluss bilden $\mathrm{Zu}$ sammenfassung und Ausblick (5).

\section{Organisations- und Kollektiv- verhandlungsstrukturen}

\subsection{Die Organisationsform der Arbeitgeber}

Die Organisation der Arbeitgeber folgt strikt dem föderalistischen Staatsaufbau von Bund, Ländern und Gemeinden bzw. Gemeindeverbänden: 
(1) Verbände im engeren Sinne existieren nur auf Ebene der Gemeinden. Die in jedem Bundesland bestehenden kommunalen Arbeitgeberverbände (KAV) schlossen sich 1949 zu einem Dachverband zusammen, der Vereinigung der kommunalen Arbeitgeberverbände (VKA), ${ }^{5}$ und beauftragten diese mit Interessenkoordination und Verhandlungsführung.

(2) Auf Ebene der Bundesländer besteht die ebenfalls 1949 gegründete Tarifgemeinschaft deutscher Länder (TdL); ${ }^{6} \mathrm{ihr}$ gehören, nachdem Berlin nach seinem 1994 erfolgten Ausschluss 2012 wieder beitrat, mit Ausnahme von Hessen, welches 2004 austrat, sämtliche Bundesländer an. Innerhalb der TdL koordinieren die Finanz- und Innenminister die Interessen der Landesregierungen.

(3) Der Bund beauftragt seit den 1960er Jahren den Bundesminister des Innern (BMI) mit der Interessenvertretung, die in Abstimmung mit anderen Ministerien sowie dem Bundeskanzleramt erfolgt. Der BMI ist zudem traditionell der Verhandlungsführer der Arbeitgeberseite.

Die Organisationsgrade, d. h. der Anteil der Organisierten an allen Arbeitgebern, sind vergleichsweise hoch, d. h. sie schöpfen ihr Mitgliederpotenzial weitestgehend aus. ${ }^{7}$ Auch die Organisationsgrade der Arbeitnehmergruppen liegen deutlich über dem gesamtwirtschaftlichen Durchschnitt. Diese Muster decken sich mit denen in der Mehrzahl vergleichbarer Länder (Hebdon/Kirkpatrick 2005; Bordogna 2008).

Die rechtlich selbstständigen KAV, die umfassende Organisationen im Sinne von Olson (1985) sind, geben autonome Rechte aus dem Kanon ihrer Tarifhoheit an die VKA $\mathrm{ab}$, ihre Spitzenvereinigung im Sinne des Tarifvertragsgesetzes; die VKA nimmt als tarifpolitischer und arbeitsrechtlicher Dachverband Koordinationsaufgaben wahr und ist für Fragen von allgemein-überregionalem Interesse zuständig. Als „Verband der Verbände“ schafft sie trotz der heterogenen Interessen ihrer Mitglieder die Voraussetzungen für kollektives Handeln und sichert die politisch gewollte Einheitlichkeit der Tarifpolitik.

Auf beiden Ebenen bestehen neben den üblichen, aus Arbeitgeberverbänden der Privatwirtschaft bekannten Gremien (vor allem Mitgliederversammlung, Haupt- oder Verhandlungsausschuss, Vorstand) auch sogenannte Gruppenausschüsse (u. a. für Verwaltung, Versorgungsbetriebe, Nahverkehr), die faktisch über weitgehende Entscheidungskompetenz und -autonomie verfügen. Diese innerverbandlich-horizontale Differenzierung nach relativ homogenen Interessen durch Dezentralisierung von Entscheidungen erleichtert die Formulierung und spätere Umsetzung arbeitsteilig-kooperativer Verbandsstrategien (Keller 2010a, 2010b).

\subsection{Strukturen der Kollektivverhandlungen}

Charakteristisch und im internationalen Vergleich (Dell'Aringa et al. 2001) ungewöhnlich ist der traditionell hohe Zentralisierungsgrad der Tarifverhandlungen, der die Existenz entsprechend komplexer Organisations- und Repräsentationsstrukturen auf beiden Seiten voraussetzt. Andere föderalistisch organisierte Staaten, vor allem die angelsächsischen (Belman et al. 1996; Bach 2010; Bach/ Bordogna 2011), sind hingegen durch weitgehende Dezentralisierung nach verschiedenen Kriterien (wie Ebene, Arbeitnehmerstatus, Funktions- bzw. Tätigkeitsbereich) gekennzeichnet. Eine Folge in der Bundesrepublik besteht in der traditionell weitgehenden Standardisierung, d.h. der politisch gewollten Herstellung und Erhaltung der Gleichheit von Arbeits- und Lebensbedingungen aller öffentlich Beschäftigten.

Die Arbeitgeber von Bund, Ländern und Gemeinden bildeten seit den 1960er Jahren eine drittelparitätisch zusammengesetzte Verhandlungsgemeinschaft. Diese enge Koordinierung führte zu weitgehender Zentralisierung: Bis 2003 fanden für die drei Ebenen stets gemeinsame Verhandlungen statt, die zu einheitlichen Abschlüssen für Arbeiter und Angestellte führten. Diese ungewöhnliche Konstellation eines ,joint bargaining“ blieb über mehrere Jahrzehnte stabil, obwohl sie aufgrund der vorhandenen latenten Interessendifferenzen erhebliche Probleme und hohen Abstimmungsbedarf im intra- und interorganisatorischen Bargaining (Walton/McKersie 1991) verursachte. Interessenunterschiede, die auch für Arbeitgeberverbände der Privatwirtschaft (u. a. durch kleine und große Mitglieder) konstitutiv sind (Behrens 2011), bestanden aufgrund der unterschiedlichen Anteile der Personal- an den Gesamtausgaben (Bund ca. $10 \%$, Länder ca. $38 \%$, Gemeinden ca. $27 \%$ ), die durch die im Grundgesetz vorgegebene Verteilung der Staatsaufgaben bedingt sind.

Aufseiten der Arbeitnehmer gibt es ebenfalls Verhandlungsgemeinschaften, weil im Gegensatz zur Privatwirtschaft traditionell „Koalitionspluralismus“ besteht. Ihre Zusammensetzung änderte sich im Zeitverlauf mehrfach; der dominierende Akteur war stets die Gewerkschaft Öffentliche Dienste, Transport und Verkehr (ÖTV) (Keller 1983, 1993). Seit 2007 verhandelt die Vereinte Dienstleistungsgewerkschaft (ver.di), die 2001 fünf Einzelgewerkschaften durch Zusammenschluss gründeten (Keller 2004), gemeinsam mit der dbb-Tarifunion, dem „Tarifflügel“ des Deutschen Beamtenbundes (DBB). ${ }^{8}$ Diese Konstellation ist insofern ungewöhnlich, als beide verschiedenen, um Mit-

\footnotetext{
5 http://www.vka.de.

6 http://www.tdl-online.de.

7 Nach der Vereinigung wurden diese etablierten Organisationsprinzipien und -strukturen auf die neuen Bundesländer übertragen (Rosdücher 1994).

8 Der DBB beschloss auf seinem Gewerkschaftstag im Herbst 2012 den Zusammenschluss der beiden bis dato getrennten Organisationseinheiten Beamtenbund und Tarifunion, http://www.dbb.de.
} 
glieder konkurrierenden Dachverbänden angehören. Die Tarifführerschaft liegt bei ver.di, die Tarifunion ist nur begrenzt streik- und durchsetzungsfähig.

Der tatsächliche, informelle Einfluss der Arbeitgeberverbände reichte mehrere Jahrzehnte weit über ihre formalrechtliche Organisationsdomäne hinaus und schuf faktisch den mit Abstand größten Tarifbereich der Bundesrepublik:

(1) Neben den „Hauptverhandlungen“ gab es bis in die 1990er Jahre stets „Nebenverhandlungen“ für Bundesbahn und Bundespost, die formal unabhängig waren, jedoch zeitlich parallel stattfanden. Sie führten, abgesehen von bereichsspezifischen Fragen (wie Zulagen), zu materiell identischen Abschlüssen, sodass die politisch gewollte Einheitlichkeit der Arbeitsbedingungen infolge dieses spezifischen Arrangements von "pattern setting" und „pattern following“ stets gewahrt blieb. Verhandlungspartner waren auf Arbeitnehmerseite die Branchenverbände bzw. Betriebsgewerkschaften, Gewerkschaft der Eisenbahner Deutschlands (GdED) und Deutsche Postgewerkschaft (DPG), auf Arbeitgeberseite die zuständigen Bundesminister. Die skizzierten Arbeitgeberverbände organisierten diese Bereiche nicht und waren daher an deren Kollektivverhandlungen nicht beteiligt. Diese Verhandlungskonstellation und die resultierende Gleichbehandlung der Beschäftigten verändern sich deutlich seit der Privatisierung der Bundesvermögen, wie im Folgenden analysiert wird.

(2) Weiterhin wurden die Ergebnisse der Kollektivverhandlungen in aller Regel zeit- und inhaltsgleich auf die Beamten übertragen, die nach herrschender Rechtslehre (Art. 33 GG) aufgrund ihres Status kein Kollektivverhandlungs- und Streikrecht haben; ihre Interessenverbände verfügen allerdings über gesetzlich garantierte Anhörungs- und Konsultationsrechte (Keller 1983, 1993). Diese enge Koppelung beider Bereiche lockert sich seit Mitte der 2000er Jahren (Keller 2010a).

(3) Die Kirchen, die nach dem öffentlichen Sektor zweitgrößten Arbeitgeber (ca. 1,3 Mio. Beschäftigte), verfügen über verfassungsrechtliche Sonderbedingungen (Art. 140 GG); der ihnen zugestandene „Dritte Weg“ schließt Tarifverhandlungen und Streiks aus. Sie richteten sich informell im Rahmen ihrer paritätisch besetzten ,arbeitsrechtlichen Kommissionen" lange Zeit nach den Ergebnissen im öffentlichen Sektor. Sie gaben diese Orientierung ihrer Einrichtungen (wie Caritas oder Diakonie, Krankenhäuser) seit Mitte der 1990er Jahre unter Wettbewerbs- und veränderten Finanzierungsbedingungen ebenfalls sukzessiv auf und handeln nun nach aus der Privatwirtschaft bekannten Maximen.

\section{Wandel der Arbeitsbeziehungen}

In den vergangenen beiden Dekaden fanden im Vergleich zu dem "goldenen Zeitalter" der bundesrepublikanischen Nachkriegsjahrzehnte erhebliche Veränderungen statt, die sowohl den Kern des öffentlichen Sektors als auch die privatisierten Bereiche, vor allem die „natürlichen Monopole" Bahn und Post, aber auch die kommunale Ebene, betreffen. $^{9}$

\subsection{Wandel im Kernbereich}

Mitte der 2000er Jahre erfolgte sowohl eine vertikale Differenzierung zwischen Bund, Ländern und Gemeinden als auch eine gewisse horizontale Differenzierung der Organisations- und Verhandlungsstrukturen auf Länderebene, die zu einer Transformation der Arbeitsbeziehungen führte.

\subsubsection{Vertikale Differenzierung}

Die mehrere Jahrzehnte bestehende Verhandlungsgemeinschaft wurde beendet; wodurch die Arbeitgeberverbände und ihre Organisationsstrukturen allerdings kaum tangiert wurden. Der Grund war der Austritt der TdL, deren Mitglieder ihre spezifischen Interessen in den internen Abstimmungsprozessen ungenügend berücksichtigt fanden und größere Autonomie sowie mehr „Flexibilität“ forderten. Seitdem finden zeitlich und inhaltlich getrennte Verhandlungen statt. Auf Bundes- und Gemeindeebene löste 2005 der Tarifvertrag für den öffentlichen Dienst (TVöD) die seit den frühen 1960er Jahren geltenden Manteltarifverträge für Arbeiter (MTArb) bzw. den Bundes-Angestelltentarifvertrag (BAT) ab.

Mittel- und langfristig führt diese veränderte Konstellation der Kollektivverhandlungen zu unterschiedlichen Ergebnissen, d. h. zu Differenzen bei Einkommen und übrigen Arbeitsbedingungen (vor allem Arbeitszeiten und Sozialleistungen); die früher von allen Akteuren gewollte „Gleichheit von Arbeits- und Lebensbedingungen aller öffentlich Beschäftigten“ wird durch eine allmählich zunehmende Differenzierung abgelöst. Unterschiede ergeben sich vor allem zwischen finanziell starken und schwachen Ländern, die nunmehr in Konkurrenz zueinander (u. a. um Lehrer und IT-Experten) treten.

\footnotetext{
9 Das Neue Steuerungsmodell - NPM, die seit den frühen 1990er Jahren diskutierte deutsche Variante von New Public Management (Naschold/Bogumil 2000) - haben nicht, wie prima facie zu vermuten wäre, die Arbeitgeberverbände entwickelt oder umgesetzt. Zuständig ist vielmehr der Deutsche Städtetag. Die eigenständige Kommunale Gemeinschaftsstelle für Verwaltungsvereinfachung (KGSt, http://www.kgst.de/home, ab 2005 Kommunale Gemeinschaftsstelle für Verwaltungsmanagement), deren Mitglied nur Gemeinden und Gemeindeverbände werden können, berät die Kommunen. Diese Arbeitsteilung entlastet vor allem die kommunalen Arbeitgeber und ihre Verbände.
} 
Insgesamt fand - ausgehend von einem im internationalen Vergleich ungewöhnlich hohen Zentralisierungsgrad - eine sukzessive Dezentralisierung der Arbeitsbeziehungen statt. Im Gegensatz zur Situation anderer, vor allem südeuropäischer EU-Mitgliedsländer (Vaughan-Whitehead 2012; European Commission 2013) hat dieser Trend seine Ursachen nicht in der aktuellen Finanz- und Schuldenkrise, sondern in früheren, unabhängigen Entwicklungen auf nationaler Ebene. ${ }^{10}$

Die Einschätzung der mittel- und langfristigen Folgen hängt vom gewählten Referenzrahmen ab: Sein Umfang ist in Relation zum skizzierten nationalen Status quo ante beachtlich. Im Vergleich der EU-Mitgliedsländer bleibt er allerdings begrenzt; die Bundesrepublik ist der Ausnahme-, nicht der Regelfall. Flächentarifverträge sind weiterhin die dominierende Form kollektivvertraglicher Regulierung. Die tariflichen Deckungsraten liegen (mit deutlich über $90 \%$ ) nach wie vor weit über dem gesamtwirtschaftlichen Durchschnitt (Beese/Vogel 2011; Bellmann et al. 2012, S. 72), werden aber durch veränderte Verhandlungsstrukturen erreicht. In der Terminologie der in der Industrial Relations-Literatur bekannten Kategorisierung (Traxler 1997, 1998) erfolgt diese Dezentralisierung in organisierter bzw. kontrollierter, nicht in wilder bzw. unkontrollierter Form. Auch die Organisationsgrade sind auf beiden Seiten nach wie vor hoch.

„Weiße Flecken der Organisationslandkarte“, wie sie aus Dienstleistungsbranchen der Privatwirtschaft bekannt sind (Behrens 2011), kommen nach wie vor nicht vor. Inzwischen sehen die Satzungen der kommunalen Arbeitgeberverbände neben den ordentlichen auch OT-Mitgliedschaften (sog. Gastmitgliedschaften) für bestimmte Bereiche ihrer Organisationsdomäne vor, besonders für kommunale Unternehmen. ${ }^{11}$ Die Eröffnung dieser Option, die seit den 1990er Jahren aus Branchen der Privatwirtschaft bekannt ist (Haipeter 2010), deutet auf abnehmende Bindungsfähigkeit bzw. -willigkeit zumindest in bestimmten Segmenten der Organisationsdomäne hin und zeigt verbandsinternen Wandel an. Sie soll Tarifflucht und Verbandsaustritte unzufriedener Mitglieder verhindern bzw. das Überleben des Verbandes sichern sowie das etablierte System der Flächen- bzw. Verbandstarifverträge stabilisieren.

Daten über die tatsächliche Nutzung dieser zusätzlichen Option liegen nicht vor. ${ }^{12}$ Eine Gründung eigenständiger
OT-Verbände, die über OT-Mitgliedschaften in bestehenden Verbänden hinausgeht, findet im öffentlichen Sektor nicht statt.

\subsubsection{Horizontale Differenzierung}

Auf Länderebene gilt ab 2006 der Tarifvertrag für den öffentlichen Dienst der Länder (TV-L). ${ }^{13}$ Hier sind zwei Besonderheiten relevant:

(1) Wie bereits erwähnt, schloss die Mitgliederversammlung der TdL 1994 das Land Berlin wegen Verstoßes gegen Satzungsgrundsätze (Zahlung einer übertariflichen Zulage im Geltungsbereich des BAT-O) aus. Die TdL verhängte damit zum einzigen Mal in ihrer Geschichte die schärfste der laut Verbandssatzung zur Verfügung stehenden Sanktionen. In der Folgezeit blieben in Berlin die alten, bundesweit geltenden Regelungen in Kraft. Erst 2003 schloss das Land einen eigenständigen Vertrag. Auf Basis einer sogenannten Eckpunktevereinbarung schlossen Senat und Gewerkschaften 2010 den in Redaktionsverhandlungen präzisierten „Tarifvertrag zur Angleichung des Tarifrechts des Landes Berlin an das Tarifrecht der Tarifgemeinschaft deutscher Länder“. Dieser „Angleichungs-TV Land Berlin“ regelt die Wiederaufnahme in die TdL mit allen Rechten und Pflichten für 2013 sowie die sukzessiv-mittelfristige Angleichung an alle geltenden Regelungen des von der TdL geschlossenen TV-L (Entgelte, Arbeitszeiten) bis 2017.

Dieser Wiedereintritt Berlins, der eine von Arbeitgeberverbänden der Privatwirtschaft im Prinzip bekannte, allerdings faktisch seltene Statusänderung in umgekehrter Richtung bedeutet, reduziert die zwischenzeitlich eingetretene horizontale Differenzierung auf Länderebene wieder und trägt zu einer gewissen Stabilisierung des Tarifvertragssystems bei. Die Gewerkschaften kommen ihrem Ziel der Wiederherstellung einheitlicher Arbeits- und Lebensbedingungen im öffentlichen Sektor einen Schritt näher.

(2) Hessen trat, wie bereits erwähnt, 2004 aufgrund andauernder Interessendifferenzen aus der TdL aus ${ }^{14}$ und agiert seitdem als unabhängige Tarifvertragspartei (single employer bargaining). Zunächst galt die Nachwirkung der gekündigten Tarifverträge, allerdings nur für bereits Beschäftigte,
10 Im Beamtenbereich erfolgt zeitlich parallel eine ähnliche Entwicklung, die andere Ursachen hat. Die 2006 in Kraft getretene Föderalismusreform I überträgt die Kompetenz zur Regelung der Beschäftigungsbedingungen der Beamten der Länder vom Bund auf die Länder (zu Einzelheiten Keller 2010a). Diese Reform macht die ebenfalls auf Betreiben der Arbeitgeber in den frühen 1970er Jahren erfolgte Zentralisierung der Zuständigkeiten rückgängig.

11 Dieser besondere Status bedeutet, Mitglied des Arbeitgeberverbands sein zu können, ohne an die geschlossenen Tarifverträge gebunden zu sein, aber die Serviceleistun- gen (im Sinne selektiver Dienstleistungen) in Anspruch nehmen zu können.

12 Daten nennt nur der KAV BW: Er hat 750 Mitglieder mit ca. 280.000 Beschäftigten und 45 Gastmitglieder, http://www. kavbw.de/Zum_Verband.

13 Zu Einzelheiten siehe Keller 2010a; Schmidt et al. 2011; für den Fall Berlin http://www.tdl-online.de unter dem Suchwort „Berlin”.

14 Gelegentlich drohen auch andere Länder (u. a. zunächst Baden-Württemberg, Niedersachsen, später Sachsen) mit Austritt, ohne jedoch ihre Drohung zu realisieren. 
nicht jedoch für Neueinstellungen, was zu Unterschieden in den Arbeitsbedingungen führte. Ab 2010 gilt der eigenständige Tarifvertrag für den öffentlichen Dienst des Landes Hessen (TV-H) ${ }^{15}$ sowie einige ergänzende Verträge.

Die korporativen Akteure beurteilen diese Situation unterschiedlich: Die CDU/FDP-Landesregierung besteht aufgrund der schwierigen Finanz- bzw. Haushaltssituation auf Beibehaltung der „eigenständigen Tariflandschaft Hessen“, die Oppositionsparteien und Gewerkschaften fordern wiederholt die Beendigung des "hessischen Sonderweges“, die Rückkehr in die TdL und damit die Übernahme des TV-L. Die weitere Entwicklung dieser horizontalen Differenzierung hängt vom Ausgang der Landtagswahl bzw. der Zusammensetzung der Koalitionsregierung ab. Ob sich durch den eigenständigen TV-H aus Sicht des Landes tatsächlich deutliche materielle Unterschiede zum TV-L (u. a. bei Entgelten, Arbeitszeiten und Sonderzahlungen) ergeben, bleibt abzuwarten. Jedenfalls ist Hessen derzeit nicht bereit, seine „exit“-Entscheidung (Hirschman 1974) zu revidieren. Anders formuliert: Die TdL kann in diesem Fall das typische Verbandsproblem der Bestandserhaltung durch dauerhafte Bindung vorhandener Mitglieder bzw. der Sicherung ihrer Loyalität nicht lösen.

Insgesamt ist das Ausmaß der vertikalen Differenzierung (zwischen Bund und Gemeinden auf der einen sowie Ländern auf der anderen Seite) deutlich größer als das der horizontalen (zwischen den Ländern). Allerdings können Unterschiede, die nicht nur bei Entgelten und Arbeitszeiten, sondern auch bei der Einstufung in Entgeltgruppen (u. a. von 200.000 angestellten Lehrern) bereits bestehen, in $\mathrm{Zu}$ kunft zunehmen.

\subsection{Wandel als Folge von Privatisierung}

Privatisierungsmaßnahmen finden seit den frühen 1980er Jahren auf sämtlichen Ebenen statt. Auf kommunaler Ebene erfolgen sie u. a. im öffentlichen Personennahverkehr, bei der Energieversorgung sowie bei Krankenhäusern. Zu den Folgen für Arbeitsbeziehungen und ihre korporativen Akteure liegen erstaunlicherweise kaum systematische quantitative Erkenntnisse vor und können in unserem Kontext nicht erstellt werden; wir konzentrieren uns im Folgenden auf die beiden bereits erwähnten großen Bereiche des ehemaligen öffentlichen Sektors.

Mitte der 1990er Jahre erfolgte die Privatisierung der ehemaligen Bundesvermögen Bahn und Post. Der Verlust ihrer Monopolstellungen bzw. die Liberalisierung ihrer vormals geschützten Märkte führte zu einer zunehmenden Heterogenisierung der Arbeitsbeziehungen. Der besondere Status der Beamten bleibt bestehen, obwohl sie in privatisierten Unternehmen tätig sind. Im Rahmen der Privatisierungen fanden erhebliche Umstrukturierungs- und Reorganisationsmaßnahmen statt, die in beiden Bereichen einen massiven, andauernden Personalabbau zur Folge haben (durch sog. natürliche Fluktuation und sozialverträgliche
Maßnahmen wie Vorruhestand und Aufhebungsverträge mit Abfindungen, aber ohne betriebsbedingte Kündigungen).

Die skizzierte frühere Sequenz von Haupt- und Nebenverhandlungen sowie die enge Koppelung ihrer materiellen Ergebnisse bestehen nicht mehr. Seit ihren Privatisierungen führen Deutsche Bahn sowie Deutsche Telekom eigenständige Kollektivverhandlungen mit den Gewerkschaften, Eisenbahn- und Verkehrsgewerkschaft (EVG) und Gewerkschaft Deutscher Lokomotivführer (GDL) bzw. ver.di, zu deren Gründungsmitgliedern die DPG gehörte (Keller 2004).

\subsubsection{Bahn}

Bei der Deutschen Bahn entstanden nach der Privatisierung neue, komplexere Verhandlungsstrukturen. Auf Arbeitnehmerseite erfolgte 2010 durch Zusammenschluss von Transnet (Transport, Service, Netze) und der Verkehrsgewerkschaft Deutscher Bundesbahnbeamten und Anwärter (GDBA) die Gründung der EVG. ${ }^{16}$ Außerdem setzte 2007/08 die Berufsgewerkschaft GDL durch längere Arbeitskampfaktionen bei der Deutschen Bahn gegen den Widerstand der Arbeitgeber ihre faktische Anerkennung als unabhängige Gewerkschaft durch und etablierte sich als autonomer Tarifpartner (Hoffmann/Schmidt 2008; Keller 2009; Kalass 2012).

Auch aufseiten der Arbeitgeber bestehen konkurrierende Verbände (Lesch 2010; Kraemer 2012), die Probleme bei der Formulierung gemeinsamer Verhandlungspositionen haben: Der Arbeitgeber- und Wirtschaftsverband Mobilität und Verkehrsdienstleistungen (AgvMoVe) ${ }^{17}$ organisiert ausschließlich die Unternehmen des Eisenbahnverkehrs und der Eisenbahninfrastruktur, die zum bundeseigenen Konzern Deutsche Bahn AG (DB AG) gehören. Der Arbeitgeberverband Deutscher Eisenbahnen (AGVDE) ${ }^{18}$ vertritt vor allem die kleineren Privatbahnen. Die Unternehmensgröße ist die entscheidende Determinante der Verbandsmitgliedschaften und -domänen, was im internationalen Vergleich keineswegs ungewöhnlich ist (Traxler et al. 2007).

Die kleineren Betreiber befürchten, bei Mitgliedschaft im größeren Verband bzw. Abschluss eines einheitlichen, echten Branchentarifvertrages ihre Konkurrenzfähigkeit einzubüßen. ${ }^{19}$

15 http://hessen.verdi.de/fachbereiche/bund-laender

16 http://www.evg-online.org, siehe auch Keller 2012.

17 http://www.agv-move.net.

18 http://www.agvde.de.

19 Im Übrigen stellen diese heterogenen Organisationsstrukturen im Vergleich der EU-Mitgliedsländer keine deutsche Besonderheit, sondern eher den Regelfall dar (Traxler/ Adam 2008). 
Zwischen EVG, DB AG und den großen privaten Bahnbetreibern (Abellio, Arriva, Benex, Keolis, Veolia Verkehr, Hessische Landesbahn) kam ein Branchentarifvertrag für alle Beschäftigten des Schienenpersonennahverkehrs (SPNV) lange Zeit nicht zustande. Die privaten „G 6-Unternehmen" wollten ihren wesentlichen Wettbewerbsvorteil, über den sie aufgrund ihrer niedrigeren Entgeltniveaus bzw. geringeren Personalkosten verfügten, nicht aus der Hand geben und weigerten sich, mit dem Branchenführer DB AG gemeinsame Verhandlungen über die Einführung gleicher Tarif- und Sozialstandards zu führen. Die DB AG hingegen hatte tendenziell Interesse an einem einheitlichen Vertrag, der die Vorteile ihrer Konkurrenten ausschalten oder zumindest minimieren würde. Warnstreiks im Herbst 2010 beeinträchtigten nicht nur den Regional-, sondern auch den Fernverkehr.

Der Anfang 2011 geschlossene, notwendigerweise komplexe Flächentarifvertrag gilt ausschließlich für den Nahverkehr; er soll einheitliche Arbeits-, insbesondere Einkommensbedingungen auf dem Niveau der DB AG schaffen bzw. einen Unterbietungswettbewerb der Verkehrsträger verhindern, der über Entgelte und Beschäftigungsbedingungen ausgetragen wird. Die Einhaltung seines (nahezu) gleichen Entgeltniveaus soll bei allen, in Zukunft zunehmenden öffentlichen Ausschreibungen und Vergaben zur Bedingung gemacht werden, um auch Nicht-Verbandsmitglieder zur Beachtung zu bewegen; die von der DB AG gegründeten Regionalgesellschaften sollen sich ebenfalls an diesen einheitlichen Branchenbedingungen orientieren. Im Übrigen fordern beide Seiten vehement, den Tarifvertrag für allgemeinverbindlich zu erklären, um eine Unterschreitung der vereinbarten Bedingungen durch die kleineren, nicht zur Gruppe der „G6-Unternehmen“ gehörenden Anbieter zu verhindern.

Den erheblichen Wandel der Strukturen der Interessenvertretung dokumentiert die bestehende Tarifpluralität. Im Nachvollzug dieser eingetretenen Entwicklungen revidierte das Bundesarbeitsgericht im Sommer 2010 das in seiner langjährigen Rechtsprechung vertretene Prinzip der Tarifeinheit endgültig und erkannte Tarifpluralismus bei konkurrierenden Verträgen an.

In Zukunft ist infolge der Liberalisierung des bisher geschützten Marktes des nationalen Schienenpersonenfernverkehrs mit mehr Konkurrenz durch private Anbieter zu rechnen, die bereits über einen eigenen Verband verfügen und unabhängige Kollektivverhandlungen führen. Die Zulassung des privaten Fernbuslinienverkehrs wird auch mehr Wettbewerb bei Entgelten und Arbeitsbedingungen zur Folge haben.

\subsubsection{Post und Telekommunikation}

Die Deutsche Telekom AG gründete 2001 den internen Arbeitgeberverband Telekom (AGV-T), dessen Mitglied sämtliche deutschen Konzernunternehmen sind (Keller 2002). Seine Verhandlungsstrategien orientieren sich an den
Prinzipien von Wettbewerb, Marktorientierung der Entgelte und intern-homogener Konditionengestaltung. Im Rahmen des 2001 eingeführten neuen Bewertungs- und Bezahlungssystems erfolgte die Abkehr von der inhaltlichen und zeitlichen Bindung an den öffentlichen Sektor ${ }^{20}$ sowie die Einführung neuer, an privatwirtschaftlichen Maximen ausgerichteten Eingruppierungs- und Entgeltregeln. „Mit der verbandsartigen Struktur wird die Zielsetzung und Umsetzung der Konditionenstrategie im Konzern unter einem Dach zusammengeführt und aus einer Hand gebündelt.“ (Welslau/Frings 2002, S. 267)

Der AGV-T nimmt nach innen (logic of membership) Aufgaben der Information, Beratung sowie Koordination wahr und fördert den Erfahrungs- und Informationsaustausch der Konzerngesellschaften; nach außen (logic of influence) vertritt er die gemeinsamen sozial- und insbesondere tarifpolitischen Interessen des Konzerns. In Verhandlungen mit den Gewerkschaften, vor allem mit ver.di, sichert er durch Abschluss von Haustarifverträgen sowie Konzern- und Gesamtbetriebsvereinbarungen unter Berücksichtigung der Unternehmensspezifika die Homogenität der Beschäftigungsbedingungen, was nicht unbedingt Einheitlichkeit bedeuten soll. Der AGV-T fungiert als Tarifvertragspartei, sodass die Tarifbindung erhalten bleibt, allerdings in anderer Form und zu veränderten Bedingungen. Er ist kein Arbeitgeberverband im üblichen Sinne, da er ausschließlich die zum Konzern gehörenden deutschen Unternehmen umfasst. Der AGV-T, der sich in Aufgabenbzw. Kompetenzbereiche gliedert, ist organisatorisch im Zentralbereich Personalmanagement angesiedelt und verfügt über keine eigene Rechtspersönlichkeit.

Die großen privaten Anbieter von Telekommunikationsleistungen (Vodafone, Telefonica, e-plus) haben sich nicht zu einem Arbeitgeberverband zusammengeschlossen. Die Vertretung ihrer gemeinsamen politischen Interessen durch Kontakte zu Ministerien und Parlament erfolgt ebenfalls ohne Verband durch informelle Koordination auf Arbeits- und zuweilen Vorstandsebene. Eine Notwendigkeit verbandlicher Organisierung wird auch in Zeiten zunehmenden Kostendrucks nicht gesehen.

Die Regelung der Arbeitsbedingungen (u. a. Entgelte, Arbeitszeit, Urlaub, Sozialleistungen, auch Einstufungen) erfolgt in Betriebsvereinbarungen mit dem Gesamtbetriebsrat bzw. den Betriebsräten, die bereits in der Gründungsphase dieser Unternehmen zustande kamen. Die Gewerkschaften ver.di, IG Metall, Industriegewerkschaft Bergbau, Chemie, Energie (IG BCE) sind - bei wesentlich niedrigeren Organisationsgraden der Betriebsräte und Mitarbeiter als bei der Telekom - nicht beteiligt; Streiks finden bei den privaten Anbietern nicht statt. Orientierungsmaßstäbe und

20 Infolge der Privatisierung änderte sich die Rechtsgrundlage der betrieblichen Interessenvertretung vom BPersVG zum BetrVG (zu Einzelheiten und Schwierigkeiten des Übergangs Wegner 2002). 
damit eine gewisse Legitimation gegenüber dem Verhandlungspartner liefern externe Personalberatungsfirmen.

\subsubsection{Folgen}

Die Beschäftigungsbedingungen bei den neuen, privaten Anbietern der ehemals öffentlich erbrachten Dienstleistungen sind noch „flexibler" und aus Arbeitnehmersicht in der Regel ungünstiger als bei den Nachfolgeunternehmen von Bahn und Post. ${ }^{21}$ Verhandlungen finden ausschließlich auf Betriebs- bzw. Unternehmensebene statt, wobei die Regelungen der Haus- und Firmentarifverträge bzw. Betriebsvereinbarungen differieren können. Die mangelnde Stabilität in prozeduraler Perspektive führt $\mathrm{zu}$ fehlender Stabilität in materieller Hinsicht: Sie ist mit einer Verschlechterung der Arbeitsbedingungen verbunden.

Insofern weisen diese Bereiche nach ihrer Privatisierung und Reorganisation deutliche Ähnlichkeiten mit Entwicklungen auf, wie sie vor allem in Dienstleistungsbranchen der Privatwirtschaft seit den 1990er Jahren stattfinden; festzustellen sind Tendenzen der Dezentralisierung, wenn nicht gar einer gewissen Fragmentierung, vor allem bei den neuen privaten Anbietern. Die Arbeitsbeziehungen sind im Gegensatz zur Praxis einer „arbeitsteiligen Kooperation“ zwischen betrieblicher und überbetrieblicher Ebene, wie sie in Privatwirtschaft und öffentlichem Sektor der Bundesrepublik traditionell üblich ist, monistisch geprägt.

Eine notwendige Voraussetzung für die Entwicklung dualer Arbeitsbeziehungen und den Abschluss von Flächentarifverträgen wäre die Gründung von Arbeitgeberverbänden mit dem Zweck der Interessenvertretung auf Branchenebene. Eine solche Absicht haben die Unternehmen jedoch nicht; allerdings bestanden auch vor den Privatisierungen keine Verbände. Die Formulierung bzw. Vertretung gemeinsamer Interessen wäre in Anbetracht der Unterschiede innerhalb der Branchen sowie der Heterogenität zwischen großen und kleineren potenziellen Mitgliedern überaus schwierig; außerdem bestünden bei wichtigen unternehmensinternen Akteuren, wie Geschäftsführungen, aus „mikropolitischen" Gründen Widerstände gegen die Abgabe von Kompetenzen, die mit einer Verbandsgründung notwendigerweise verbunden wäre. Schließlich können die Anbieter aufgrund ihrer geringen Zahl ihre Interessen auch ohne Plattform eines Verbandes informell abstimmen sowie nach außen vertreten, falls sie eine derartige Koordination für sinnvoll bzw. notwendig halten.

Insofern besteht das aus der Verbandstheorie und -forschung bekannte, bei großen Gruppen im Prinzip schwierig zu lösende Kollektivgutproblem bzw. die Verhinderung von Trittbrettfahren kaum. Stattdessen existieren in Bezug auf Handlungsfähigkeit die typischen Vorteile kleiner Gruppen (Olson 1968, 1985), die kaum eine verbandsförmige Organisation zur wirksamen Vertretung ihrer Interessen benötigen.

Die ehemaligen Gewerkschaften in diesen Bereichen, DPG und GdED bzw. Transnet, waren nie, wie in der Bundesrepublik zumeist üblich, Industrie-, sondern Betriebs- gewerkschaften. Ihren Nachfolgern gelingt das zentrale Anliegen sektoraler Tarifpolitik, „to take wages out of competition“, nicht mehr, jedenfalls nicht wie früher für die gesamte Branche, sondern allenfalls für einzelne Unternehmen. Infolge der Etablierung von mehr und kleineren Verhandlungseinheiten steigen ihre Transaktions- im Sinne von Koordinations- und Verhandlungskosten (Williamson 1985, 1996) und erfordern den vermehrten Einsatz knapper personeller und zeitlicher Ressourcen; zudem besteht zunehmende Gewerkschaftskonkurrenz, die eine Koordinierung von Interessen erschwert.

Im Übrigen sind die Organisationsgrade der Arbeitnehmer, ein wichtiger Indikator für Mobilisierungspotenzial und Durchsetzungsfähigkeit, bei den privaten Anbietern deutlich niedriger als bei Telekom und DB AG. Damit sind die für die Vertretung der Mitgliederinteressen bzw. die Zielerreichung der Organisation notwendigen materiellen Ressourcen begrenzt (Pfeffer/Salancik 2003).

\section{Schluss}

Ein Zerfall der Arbeitgeberverbände findet im Kernbereich des öffentlichen Sektors ebenso wenig statt wie eine Erosion der Flächentarifverträge im Sinne einer fortschreitenden Verbetrieblichung mit mehr und kleineren Verhandlungseinheiten; die Dezentralisierung hat organisierten bzw. kontrollierten, nicht wilden bzw. unkontrollierten Charakter. In der Privatwirtschaft verbreitete Phänomene wie Verbandsflucht oder Verbandsabstinenz haben keine Pendants. Die im Vergleich zur Privatwirtschaft weit überdurchschnittlichen tariflichen Deckungsraten bleiben bei erheblich veränderten Organisations- und Verhandlungsstrukturen bestehen.

Unser Fazit lautet, dass es sich nach wie vor um weitgehende Repräsentationsmonopole bzw. um relative institutionelle Stabilität bei oder gar trotz sozio-ökonomischem Wandel handelt; Letzterer findet vor allem, aber nicht ausschließlich in den privatisierten Bereichen von Bahn und Post statt. Deutlich abgenommen hat der bis in die 1990er Jahre erhebliche informelle Einfluss der Arbeitgeberverbände, der, wie eingangs skizziert, nicht nur weit über ihre Organisationsdomänen, sondern sogar über den öffentlichen Sektor hinausreichte.

Öffentliche Arbeitgeber sind längst nicht mehr „Modellarbeitgeber“ (Bach et al. 2009), die sie in den

21 Auf kommunaler Ebene bestehen nach Privatisierungen häufig Sparten-Tarifverträge. Sie weisen durch ihre stärkere Orientierung an Wettbewerbsbedingungen bzw. durch Versuche der Kosten-, insbesondere Arbeitskostensenkung eher Parallelen zur Privatwirtschaft als zum öffentlichen Sektor auf. Zu Einzelheiten aus dem Bereich des ÖPNV siehe Wendl 1998. 
Nachkriegsjahrzehnten auch für die Privatwirtschaft waren. Sie orientieren sich stärker an betriebswirtschaftlichen Kostenkalkülen; die früher erheblichen Unterschiede zur Privatwirtschaft nehmen ab (Keller/Henneberger 1999), wobei dieser Trend in den 2000er Jahren deutlicher wurde. Beschäftigungssicherheit ist nicht mehr stets und überall ein zentrales Merkmal der Arbeitsbedingungen.

Ungeklärt bleiben müssen derzeit die mittel- und langfristigen Folgen zunehmender Haushaltsdefizite und öffentlicher Verschuldung, anhaltender Austeritätspolitik und möglichen konjunkturellen Abschwungs (Keller 2013a). Massive Konsequenzen sind vor allem in südeuropäischen Ländern zu beobachten, nicht hingegen in Skandinavien oder Deutschland (European Commission 2013). Diese ungleiche Verteilung kann sich im Verlauf der andauernden Finanz- und Schuldenkrise sowie der Umsetzung der zunächst national inzwischen auch auf EU-Ebene vereinbarten Schuldenbremse ändern. Deren strikte Vorgaben in Bezug auf Haushaltskonsolidierung reichen weit über die Beschäftigten hinaus, deren Konfliktbereitschaft zunehmen kann, und treffen große Gruppen von Bürgern.

\section{LITERATUR}

Bach, St. (2010): Public sector industrial relations: The challenge of modernization, in: Colling, T./Terry, M. (Hrsg.): Industrial relations. Theory and practice, Chichester, S. 151-177

Bach, St./Bordogna, L. (Hrsg.) (2011): Special issue: Varieties of new public management? Public service employment relations in transition, in: International Journal of Human Resource Management 22 (11), S. 2281-2383

Bach, St./Bordogna, L./Della Rocca, G./Winchester, D.(Hrsg.) (1999): Public service employment relations in Europe. Transformation, modernization or inertia, London

Bach, St./Kolins Givan, R./Forth, J. (2009): The public sector in transition, in: Brown, W./Bryson, A./Forth, J./Whitfield, K. (Hrsg.): The evolution of the modern workplace, Cambridge/New York, S. 307-331

Beese, B./Vogel, S. (2011): Germany: Representativeness study of the European social partners organisations - public administration sector, http://www. eurofound.europa.eu/eiro/studies/tn0912027s/de0912029q.htm

Behrens, M. (2011): Das Paradox der Arbeitgeberverbände. Von der Schwierig keit, durchsetzungsstarke Unternehmensinteressen kollektiv zu vertreten, Berlin Bellmann, L. et al. (2012): Entwicklungen und Perspektiven des deutschen Arbeitsmarktes, in: Brücker, H./Klinger, S./Möller, J./Walwei, U. (Hrsg.): Handbuch Arbeitsmarkt 2013. Analysen, Daten, Fakten, Nürnberg/Bielefeld, S. 13-92

Belman, D./Gunderson, M./Hyatt, D. (Hrsg.) (1996): Public sector employment in a time of transition, Madison

Bordogna, L. (2008): Moral hazard, transaction costs and the reform of public service employment relations, in: European Journal of Industrial Relations 14 (4), S. $381-400$

Dell'Aringa, C./Della Rocca, G./Keller, B. (Hrsg.) (2001): Strategic choices in reforming public service employment. An international handbook, Houndmills/ New York

European Commission/Directorate-General for Employment, Social Affairs and Inclusion (2013): Industrial relations in Europe 2012, Commission Staff Working Document, Brussels

European Foundation for the Improvement of Living and Working Conditions (2011): Representativeness of the European social partner organization: Public administration, Dublin

Haipeter, Th. (2010): OT-Mitgliedschaften und OT-Verbände, in: Schroeder, W./ Weßels, B. (Hrsg.): Handbuch Arbeitgeber- und Wirtschaftsverbände in Deutschland, Wiesbaden, S. 209-219

Hebdon, R./Kirkpatrick, I. (2005): Changes in the organization of public services and their effects on employment relations, in: Ackroyd, St./Batt, R./ Thompson, P./Tolbert, P.S. (Hrsg.): The Oxford handbook of work and organization, Oxford, S. 531-553
Hirschman, A. O. (1974): Abwanderung und Widerspruch. Reaktionen auf Leistungsabfall bei Unternehmungen, Organisationen und Staaten, Tübingen Hoffmann, J./Schmidt, R. (2008): Der Streik der Lokführer-Gewerkschaft GDL. Anfang vom Ende des deutschen Systems der industriellen Beziehungen?, in: PROKLA. Zeitschrift für kritische Sozialwissenschaft 38 (151), S. 323-342

Kalass, V. (2012): Neue Gewerkschaftskonkurrenz im Bahnwesen. Konflikt um die Gewerkschaft Deutscher Lokomotivführer, Wiesbaden

Keller, B. (1983): Arbeitsbeziehungen im öffentlichen Dienst. Tarifpolitik der Gewerkschaften und Interessenpolitik der Beamtenverbände, Frankfurt/New York

Keller, B. (1993): Arbeitspolitik des öffentlichen Sektors, Baden-Baden

Keller, B. (2002): Ver.di: Von der Industrie - zur Multibranchengewerkschaft, in: Klinkhammer, H. (Hrsg.): Personalstrategie. Personalmanagement als Business Partner, Neuwied/Kriftel, S. 282-306

Keller, B. (2004): Multibranchengewerkschaft als Erfolgsmodell? Zusammenschlüsse als organisatorisches Novum - das Beispiel ver.di, Hamburg

Keller, B. (2009): Berufs- und Spartengewerkschaften: Konsequenzen und Optionen, in: Sozialer Fortschritt 59 (6), S. 118-128

Keller, B. (2010a): Arbeitspolitik im öffentlichen Dienst. Ein Überblick über Arbeitsmärkte und Arbeitsbeziehungen, Berlin

Keller, B. (2010b): Arbeitgeberverbände des öffentlichen Sektors, in: Schroeder, W./Weßels, B. (Hrsg.): Handbuch Arbeitgeber- und Wirtschaftsverbände in Deutschland, Wiesbaden, S. 105-125

Keller, B. (2012): Der Zusammenschluss von Gewerkschaften im Eisenbahnsektor - von Vernunftehen und unwilligen Dritten, in: Arbeit. Zeitschrift für Arbeitsforschung, Arbeitsgestaltung und Arbeitspolitik 21 (4), S. 247-262

Keller, B. (2013a): Der öffentliche Dienst in der Finanz- und Schuldenkrise. Austeritätspolitik und die Entwicklung der Arbeitsbeziehungen, in: Sozialer Fortschritt 62 (im Druck)

Keller, B. (2013b): Gewerkschaften und Interessenverbände im System der Arbeitsbeziehungen des öffentlichen Sektors, in: Schroeder, W./Weßels, B. (Hrsg.): Die Gewerkschaften in Politik und Gesellschaft der Bundesrepublik Deutschland, 2. Aufl., Wiesbaden (im Druck)

Keller, B./Henneberger, F. (1999): Privatwirtschaft und öffentlicher Dienst: Parallelen und Differenzen in den Arbeitspolitiken, in: Müller-Jentsch, W. (Hrsg.): Konfliktpartnerschaft. Akteure und Institutionen der industriellen Beziehungen, München/Mering, S. 233-256

Keller, B./Schnell, R. (2003): Zur empirischen Analyse von Personalräten Strukturdaten und Probleme der Interessenvertretung, in: WSI-Mitteilungen 56 (3), S. 185-193, http://www.boeckler.de/cps/rde/xbcr/hbs/wsimit_2003_03_ keller.pdf

Keller, B./Schnell, R. (2005): Sozialstruktur und Problemfelder der Interessenvertretung im öffentlichen Dienst. Eine empirische Untersuchung von Personalräten in West- und Ostdeutschland, in: Berliner Journal für Soziologie 15 (1), S. 87-102

Kraemer, B. (2012): Employment and industrial relations in the railways sector http://www.eurofound.europa.eu/eiro/studies/tn1109030s/de1109039q.htm Lesch, H. (2010): Bahn-Tarifverhandlungen Interessenvielfalt, http://www.iwkoeln.de/Publikationen/Gewerkschaftsspiegel/Archiv/tabid/135/articleid/30475/ language/de-DE/Default.aspx

McPherson, W. H. (1971): Public employee relations in West Germany: University of Michigan-Wayne State University, Institute of Labor and Industrial Relations, Ann Arbor

Naschold, F./Bogumil, J. (2000): Modernisierung des Staates. New Public Management in deutscher und internationaler Perspektive, Opladen

Olson, M. (1968): Die Logik des kollektiven Handelns, Tübingen

Olson, M. (1985): Aufstieg und Niedergang von Nationen. Ökonomisches Wachstum, Stagflation und soziale Starrheit, Tübingen

Pfeffer, J./Salancik, G. (2003): The external control of organizations, Stanford Rosdücher, J. (1994): Kommunale Arbeitgeberverbände in den neuen Bundesländern, in: Zeitschrift für öffentliche und gemeinwirtschaftliche Unternehmen 17 (4), S. 414-429

Schmidt, W./Müller, A./Trittel, N. (2011): Der Konflikt um die Tarifreform des öffentlichen Dienstes. Verhandlungsprozesse und Umsetzungspraxis, Berlin Schroeder, W./Weßels, B. (Hrsg.) (2010): Handbuch Arbeitgeber- und Wirtschaftsverbände in Deutschland, Wiesbaden

Traxler, F. (1997): Der Flächentarifvertrag in der OECD. Entwicklung, Bestandsbedingungen und Effekte, in: Industrielle Beziehungen 4 (2), S. 101-124 Traxler, F. (1998): Collective bargaining in the OECD: Developments, preconditions and effects, in: European Journal of Industrial Relations 4 (2), S. $207-$ 226 
Traxler, F./Adam, G. (2008): Representativeness of the European social partner organizations: Railways sector, http://www.eurofound.europa.eu/eiro/studies/ tn0710037s/tn0710037s_1.htm

Traxler, F./Brandl, B./Pernicka, S. (2007): Business associability, activities and governance: cross-national findings, in: Traxler, F./Huemer, G. (Hrsg.): Handbook of business interest associations, firm size and governance. A comparative analytical approach, London/New York, S. 351-406

Vaughan-Whitehead, D. (Hrsg.) (2012): Public sector adjustments in Europe: Scope, effects and policy issues, Geneva

Vereinigung der kommunalen Arbeitgeberverbände (VKA) (2012): Personalbestand steigt, in: VKA-Nachrichten 5/2012, S. 12

Vesper, S. (2012): Finanzpolitische Entwicklungstendenzen und Perspektiven des Öffentlichen Dienstes in Deutschland. Gutachten im Auftrag des Instituts für Makroökonomie und Konjunkturforschung (IMK) in der Hans-Böckler-Stiftung, Berlin

Walton, R. E./McKersie, Robert B. (1991): A behavioral theory of labor negotiations. An analysis of a social interaction system, New York

Wegner, W. (2002): Betriebliche Mitbestimmung: Sozialpartnerschaft in der Deutschen Telekom AG, in: Klinkhammer, H. (Hrsg.): Personalstrategie. Personalmanagement als Business Partner, Neuwied/Kriftel, S. 307-319

Welslau, D./Frings, D. (2002): Marktorientierung und homogene Konditionsgestaltung im Konzern, in: Klinkhammer, H. (Hrsg.): Personalstrategie. Personalmanagement als Business Partner, Neuwied/Kriftel, S. 267-281
Wendl, M. (1998): Konkurrenz erzwingt Absenkung. Die Erosion der Flächentarifverträge des öffentlichen Dienstes am Beispiel des Personennahverkehrs, in: König, O./Stamm, S./Wendl, M. (Hrsg.): Erosion oder Erneuerung? Krise und Reform des Flächentarifvertrags, Hamburg, S. 100-115

Williamson, O. E. (1985): The economic institutions of capitalism. Firms, markets, relational contracting, New York/London

Williamson, O. E. (1996): The mechanisms of governance, Oxford

\section{AUTOR}

BERNDT KELLER, Prof. Dr., war bis 2009 Professor für Arbeits- und Sozialpolitik an der Universität Konstanz. Arbeitsschwerpunkte: Arbeitspolitik des öffentlichen Sektors, Folgeprobleme der europäischen Integration, atypische Beschäftigungsverhältnisse, Flexicurity, Zusammenschlüsse von Gewerkschaften

berndt.karl.keller@uni-konstanz.de 